\title{
CyberKnife Boost in Pediatric Ependymoma of the Fourth Ventricle
}

\author{
Amr Mousa 1, 2, Yasser Khafaga 1, 2, Mohamed N. Elbeltagi ${ }^{2,3}$, Abdullah M. Al Kafi ${ }^{4}$, \\ Amani Al-Kofide 5, 6 , Ahmed Elashwah 1, 7
}

${ }^{1}$ Oncology Center, King Faisal Specialist Hospital and Research Center, Riyadh, Saudi Arabia; ${ }^{2}$ Radiation Oncology Department, National Cancer Institute, Cairo University, Cairo, Egypt; ${ }^{3}$ St. Luke's Radiation Oncology Network, Radiation Oncology, Dublin, Ireland; ${ }^{4}$ Biomedical Physics Department, King Faisal Specialist Hospital and Research Center, Riyadh, Saudi Arabia; ${ }^{5}$ Department of Pediatric Hematology/ Oncology King Faisal Specialist Hospital and Research Center, Riyadh, Saudi Arabia; ${ }^{6}$ Faculty of Medicine, Al-Faisal University, Riyadh, Saudi Arabia; ${ }^{7}$ Kasr Al-Ainy Center of Clinical Oncology and Nuclear Medicine (NEMROCK), Kasr Al-Ainy School of Medicine, Cairo University, Cairo, Egypt

Background: Ependymoma of the fourth ventricle is a challenging disease. Brain stem tolerance dose usually hinders the delivery of adequate radiation dose to target volumes.

Aim: To present the treatment outcome of pediatric ependymoma of the fourth ventricle using combined CyberKnife and volumetric modulated arc therapy (VMAT) and compare it to VMAT plans.

Methods: The medical charts of 15 patients were retrospectively reviewed. All patients underwent surgery and received radiotherapy in 2 phases using combined plans of VMAT and CyberKnife boost aiming for a total prescription dose of 59.4 Gy in 33 fractions. These plans were compared to a cone-down two-phase VMAT plans. At least $95 \%$ of the target volume was required to be encompassed by the $95 \%$ isodose level of prescription dose.

Results: It was not feasible to achieve adequate target coverage using VMAT without exceeding the brain stem tolerance doses in all but one case. Brain stem maximum point dose, V59 Gy, V55.8 Gy and mean doses were $60.46 \pm 0.3 \mathrm{~Gy}, 1.62 \pm$ $0.42 \mathrm{cc}, 37.49 \pm 5.78 \%$ and $54.79 \pm 0.64 \mathrm{~Gy}$ in VMAT plans compared to $59.67 \pm 0.21 \mathrm{~Gy}, 0.55 \pm 0.22 \mathrm{cc}, 25.49 \pm 3.84 \%$ and $52.86 \pm 0.88 \mathrm{~Gy}$ in combined technique plans $(\mathrm{p}=0.002,0.001,0.001$ and 0.001 ; respectively). The estimated 5-year progression-free and overall survival rates were $53.3 \%$ and $63.6 \%$. Performance status and extent of surgery significantly influenced overall survival. None of the patients had serious toxicities.

Conclusion: On the contrary to VMAT cone-down plans, it was possible to achieve adequate target coverage without violating brain stem constraints using the combination of VMAT and CyberKnife techniques in pediatric ependymoma of the fourth ventricle. The treatment protocol was well tolerated with no reported serious radiation toxicities.

Keywords: CyberKnife, Ependymoma, Fourth ventricle, Pediatric, Volumetric modulated arc therapy

Corresponding Author: Amr Mousa, MD; Oncology Center, King Faisal Specialist Hospital and Research Center, Riyadh, Saudi Arabia; Email: amrgabermousa@yahoo.com

Submitted: 5-May-2020, Revised: 23-May-2020, Accepted: 1-June-2020, Published online: 9-June-2020

\section{INTRODUCTION}

Ependymoma is the $3^{\text {rd }}$ most frequent pediatric brain tumor, representing $6-10 \%$ of intracranial tumors ${ }^{1,2}$. It usually arises from the ependymal cell lining of the ventricles with $90 \%$ occurrence in the cranium. More than $70 \%$ of intracranial ependymomas are located in the posterior fossa $(\mathrm{PF})^{3,4}$.

Treatment strategies for localized infratentorial ependymomas are focused on maximizing local tumor control ${ }^{5-10}$. Progression-free survival (PFS) and overall survival (OS) rates for intracranial ependymomas are not satisfactory. Five-year PFS and OS rates range from $39 \%$ to $74 \%$ and from 64 to $85 \%$, respectively $5,11,12$. Multivariate analysis showed significantly improved OS with gross total resection (GTR) and post-operative radiotherapy (RT) ${ }^{12}$. Recently, genomic and epigenomic studies have classified ependymoma into distinct molecular subgroups with different prognoses 13-15. Despite therapy, the majority of relapses occur at the local tumor site ${ }^{12}$. Delivery of adequate radiation dose to the PF ependymoma is usually hindered by the tolerance of adjacent critical structures.

In this work, we are presenting our experience in the treatment of pediatric fourth ventricular ependymoma. A combined volumetric modulated arc therapy (VMAT) and CyberKnife plans were used for the treatment of such patients. This combined technique was compared to VMAT cone-down plans.

\section{METHODS}

This retrospective study included pediatric patients with non-metastatic ependymoma originating from the fourth ventricle with the primary tumor in close proximity $(<5 \mathrm{~mm})$ to the brain stem. The study period was from 
2011 to 2017 and included eligible patients treated at the Radiation Oncology Unit of King Faisal Specialist Hospital \& Research Center (KFSH \& RC), Riyadh.

All patients had preoperative magnetic resonance imaging (MRI) scan of the whole craniospinal axis. All patients had maximum safe surgical resection prior to RT. Lumbar puncture for cerebrospinal fluid cytological analysis was done 10-14 days after surgery. Pros and cons of RT were clearly discussed with the patients' guardians and informed consents were signed. For young patients requiring general anesthesia (GA), a separate GA consent was signed. Post-operative RT started within $6 \pm$ 3.6 weeks from the date of surgery.

\section{Radiotherapy}

When needed because of young age, our GA protocol for pediatric cases undergoing daily radiation treatment adopts continuous intravenous propofol infusion providing adequate sedation without intubation. The details of our sedation protocol were published earlier ${ }^{16}$.

Computerized tomography (CT) scan simulation was done in the supine position, with the head of the patient immobilized in a neutral neck position and using a custom-molded thermoplast (Posifix ${ }^{\mathrm{TM}}$ ). Low artifactradiopaque markers required for VMAT planning were placed on the thermoplast prior to planning.

Phase I treatment: The planning CT dataset, as well as the pre- and post-operative brain MRI scans, were pushed to Eclipse $\mathrm{T}^{\mathrm{TM}}$ treatment planning system. These three datasets were fused and contouring of different volumes and organs at risk (OAR) was done. Gross tumor volume $(\mathrm{GTVi})$ included all residual tumor and/or the tumor bed as per post-operative/pre-irradiation neuroimages (pre-operative images were used only for guidance). Clinical target volume (CTVi) included the GTVi with an added $10 \mathrm{~mm}$ margin for consideration of any subclinical microscopic disease. Planning target volume (PTVi) was created by an isotropic expansion of the CTVi with a margin of $3 \mathrm{~mm}$. All patients received 50.4 Gy in 28 fractions. The VMAT plan used complete 360-degrees co-planar arcs or hemi-arcs in clockwise and counter clockwise directions. All VMAT plans were created with 6-MV flattening filter free X-rays. Treatment plans were inversely planned with Varian ${ }^{\mathrm{TM}}$ Eclipse treatment planning system (TPS) and optimized using analytical anisotropic algorithm. Plans were normalized for PTVi to be covered with at least $95 \%$ of the prescribed dose for phase I. Overlapping volume of the brain stem (BSi) and spinal cord included in the PTVi was intentionally meant to be in the relatively low dose coverage area $(95-100 \%)$.

Phase II treatment: The process of boost planning is complex and takes around 10 days. Therefore, phase II planning was started after the 3rd week of RT to avoid delay in delivering phase II treatment.

Planning CT simulation data set and a new thin-sliced (every $1 \mathrm{~mm}$ ) MRI brain scan were pushed to Accuray ${ }^{\mathrm{TM}}$ CyberKnife and Varian ${ }^{\mathrm{TM}}$ Eclipse TPS. Contouring of boost GTV (GTVii) included all residual tumor and/or the collapsed tumor bed as shown in the boost planning MRI images. Boost CTV (CTVii) in all cases included the GTVii with an additional $5 \mathrm{~mm}$ margin in all directions except in the anterior direction where the added margin was limited to $3 \mathrm{~mm}$ only. In VMAT plans, PTVii was created by isotropic expansion of the CTVii by $3 \mathrm{~mm}$. Using conventional fractionation (1.8 Gy per fraction), 9 Gy in 5 fractions was prescribed to PTVii and CTVii in VMAT and CyberKnife plans, respectively. Brain stem (BSii) was re-contoured based on the latest MRI images. Again, maximum point dose to the overlapping volume between CTVii or PTVii with the BSii or spinal cord was not allowed to exceed $100 \%$ of the prescription dose (PD).

Sum plan: For the combined technique plan, phase-I VMAT and CyberKnife boost plans were pushed to Velocity ${ }^{\mathrm{TM}}$ platform software to review their sum plan. On the other side, plans of phase I and II VMAT plans were summed on Eclipse TPS. Our acceptance criteria entailed that at least $95 \%$ of CTVii (in combined technique plans) and PTVii (in VMAT plans) to be encompassed within the $95 \%$ isodose shell without exceeding the brain stem tolerance doses. In case it was not possible in one plan, we attained the requested coverage keeping the dose to the brain stem as low as possible for comparative purposes. In our acceptance criteria, no more than $10 \%$ of the CTVii (in combined plans) and PTVii (in VMAT plans) should receive > $110 \%$ of the PD. We aimed to keep brain stem maximum point dose < $60 \mathrm{~Gy}$, V59 Gy less than $1 \mathrm{cc}$. and V55.8 Gy less than $1 / 3$ of the brain stem volume. Mean brain stem dose should be $<54 \mathrm{~Gy}$. Whenever included in the treated volumes, spinal cord V55 Gy should be $<1 \mathrm{cc}$ and maximum point dose $<55.8$ Gy in 33 fractions. Cochlear maximum dose was kept below 45 Gy in 33 fractions.

Follow up: The Common Toxicity Criteria version $2.0{ }^{17}$ was used during course of RT to evaluate toxicities. Patients were assessed in the neurosurgery and radiation oncology clinics with follow up brain MRI scan every 46 months for the first 2 years and every 6 months thereafter. An MRI spine scan was done only if clinically indicated.

\section{Ethical approval}

The study was approved by the Institutional Review Board of King Faisal Specialist Hospital and Research Center, Riyadh, Saudi Arabia (approval \# 2121030).

\section{Statistical methods}

Data analysis was performed using the Statistical Package for Social Sciences (SPSS) vs.18.

Numerical data were summarized using means $( \pm$ standard deviations) and were explored for normality using Kolmogorov-Smirnov and Shapiro-Wilk tests. The Mann-Whitney test was used to compare between the 2 groups (Combined technique versus VMAT). The Kaplan-Meier method was used to estimate PFS and OS. Linear regression model was used to identify predictors of survival.

All p-values were two-sided and those $<0.05$ were considered significant. 


\section{RESULTS}

Fifteen patients were included in this work. Their median age was 5.6 years (range: $2-13$ years) and 10/15 $(66.7 \%)$ were males.

The extent of surgery was GTR in $5(33.3 \%)$ patients, near total resection (>90\% resection of tumor volume) in $2(13.3 \%)$, debulking in $7(46.7 \%)$ and biopsy in $1(6.7 \%)$. According to the WHO grading system $2016^{18}, 4$ (26.7\%) patients had grade III and $11(73.3 \%)$ had grade II. No molecular classification was done in this group of patients. Six $(40 \%)$ patients had ventricular shunt insertion for obstructive hydrocephaly (4 preoperatively and 2 post resection). Lansky-play performance scale ${ }^{19}$ was 100 in 3 (20\%) patients, 80-90 in 4 (26.7\%), 60-70 in $5(33.3 \%)$ and $40-50$ in $3(20 \%)$.

Four out of the 15 (26.7\%) patients received RT under GA. All patients received a total dose of 59.4Gy/33 fractions using combined VMAT/CyberKnife plans. An average overall treatment time for the whole course was 49.2 \pm 1.5 days. Mean GTVi, CTVi and PTVi volumes were: $22.8 \pm 4.3, \quad 96.8 \pm 9.1$ and $168.7 \pm 12.4 \mathrm{cc}$, respectively. GTV volume was reduced to an average of $8.8 \pm 1.7 \mathrm{cc}$ in GTVii as a consequence of further collapse of the tumor bed cavity. On average, CTVii and PTVii volumes were found to be $26.79 \pm 2.3$ and $42.15 \pm 5.2 \mathrm{cc}$, respectively. VMAT plans were delivered via 2 complete arcs. Phase II course on the CyberKnife was delivered via a mean of $113 \pm 49.5$ beamlets.

Respecting brain stem constraints, the mean CTVii dose was $59.36 \pm 0.2$ Gy in the combined modality sum plans (Table 1). The average maximum point dose was $63.72 \pm 0.7 .1$ Gy $(107.28 \% \pm 1.2)$. In the combined modality arm, the $95 \%$ isodose shell encompassed $95.09 \% \pm 0.04$ of target (CTVii). Achieving the same target (PTVii) coverage in VMAT plans was not possible without exceeding the brain stem tolerance doses in all but 1 case. For comparative purpose, VMAT sum plans were run to achieve the same coverage and keeping the brain stem and/or spinal cord doses as low as possible (Figures 1A and 1B).
Table 1: Comparison of means of target volumes coverage in both sum plans

\begin{tabular}{llll}
\hline Parameter & $\begin{array}{l}\text { PTVii } \\
\text { (VMAT) }\end{array}$ & $\begin{array}{l}\text { CTVii } \\
\text { (combined } \\
\text { modality) }\end{array}$ & $\begin{array}{l}\text { p } \\
\text { value }\end{array}$ \\
\hline Volume (cc) & $42.15 \pm 5.22$ & $26.79 \pm 2.3$ & 0.001 \\
\hline Mean dose (Gy) & $59.29 \pm 0.23$ & $59.36 \pm 0.2$ & 0.143 \\
\hline V95 (\%) & $95.06 \pm 0.05$ & $95.09 \pm 0.04$ & 0.234 \\
\hline V90 (\%) & $98.79 \pm 0.42$ & $98.83 \pm 0.3$ & 0.810 \\
\hline $\begin{array}{l}\text { Maximum dose } \\
(\%)\end{array}$ & $105.75 \pm$ & $107.28 \pm 1.2$ & 0.001 \\
\hline
\end{tabular}

CTV: Clinical target volume, PTV: Planning target volume, VMAT: volumetric modulated arc therapy

Maximum, mean, V55.8 Gy and V59 Gy of the brain stem were found to be significantly lower using CyberKnife boost technique than the VMAT plan (Table 2).

Total doses calculated at the brain stem volume contoured at initial planning phase (BSi), guided by the immediate post-operative MRI scans, were significantly less than the doses calculated at the brain stem volume contoured after further collapse of the tumor bed cavity at boost phase (BSii) (Table 3).

In the 4 patients where the disease extended down to the $\mathrm{C} 1-2$ spinal cord level, the maximum point dose of the spinal cord was $55.74 \pm 0.8 \mathrm{~Gy}$ and $54.54 \pm 0.4 \mathrm{~Gy}$ in the VMAT and combined plans, respectively.

After a median follow-up period of 51 months (range 30 - 89 months), 7 (46.7\%) patients had disease progression (DP). Two patients progressed solely at the $\mathrm{PF}$ and one relapsed at the spine in addition to the PF with a local control rate of $80 \%$. The remaining 4 relapsing patients developed spinal and supra-tentorial disease with an overall estimated 5-year PFS of 53.3\%. The median time for DP was 26.6 months (range 13 - 55 months). Among different clinical parameters, patients having less than near total resection had a tendency for DP $(\mathrm{p}=0.055)$ (Table 4).

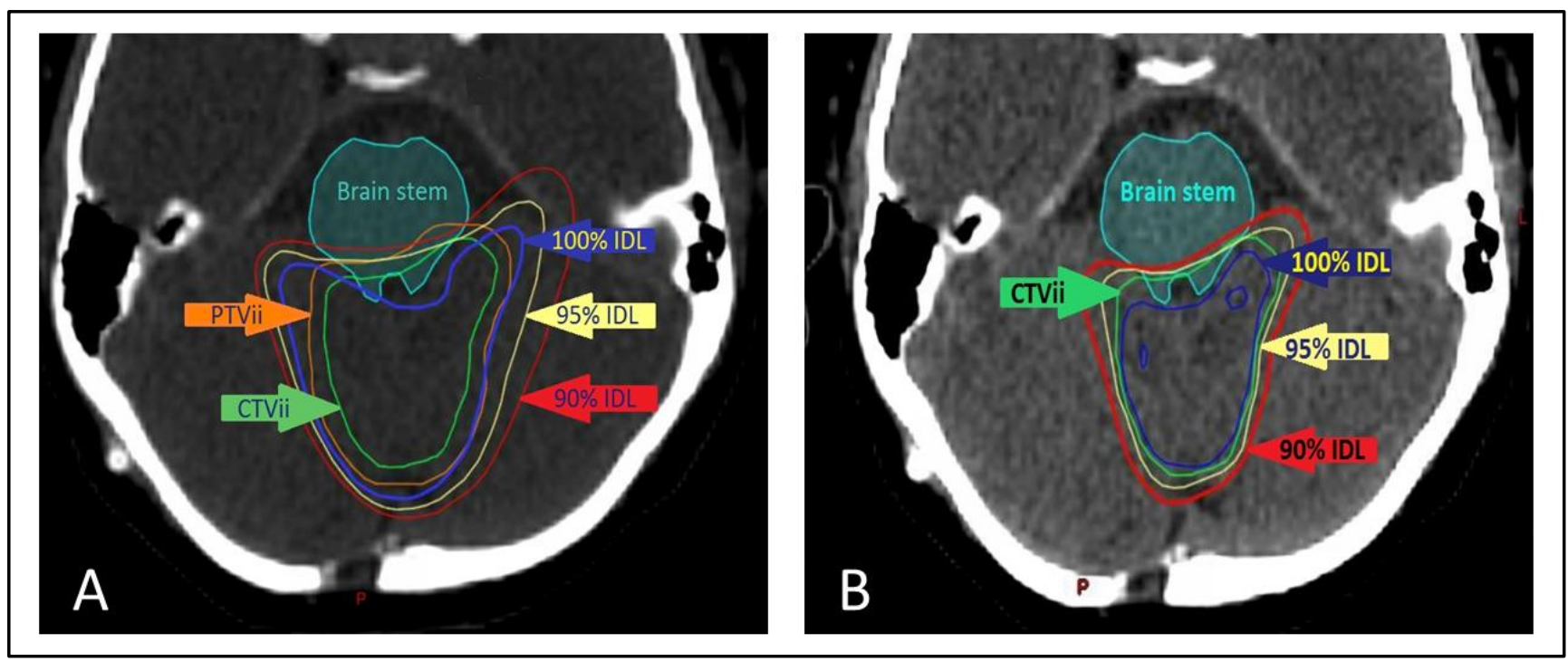

Figure 1: Isodose lines distribution of sum plans (59.4 Gy / 33 fractions) using (A) VMAT (B) Combined VMATCyberKnife 
Table 2: Comparison of mean of doses calculated at organs at risk in both sum plans

\begin{tabular}{lllll}
\hline Organ & Parameter & VMAT plans & Combined VMAT-CyberKnife plans & p value \\
\hline \multirow{2}{*}{ Brain stem } & Maximum (Gy) & $60.46 \pm 0.3$ & $59.67 \pm 0.21$ & 0.002 \\
\cline { 2 - 4 } & V59 Gy (cc) & $1.62 \pm 0.42$ & $0.55 \pm 0.22$ & 0.001 \\
\cline { 2 - 4 } & V55.8 Gy (\%) & $37.49 \pm 5.78$ & $25.49 \pm 3.84$ & 0.001 \\
\cline { 2 - 5 } & Mean (Gy) & $54.79 \pm 0.64$ & $52.86 \pm 0.88$ & 0.001 \\
\hline \multirow{2}{*}{ Optic Chiasm } & Mean (Gy) & $19.86 \pm 6.9$ & $19.76 \pm 7.1$ & 0.668 \\
\cline { 2 - 5 } & Maximum (Gy) & $26.74 \pm 7.7$ & $27.13 \pm 7$ & 0.311 \\
\hline Right Optic nerve & Mean (Gy) & $10.83 \pm 5.7$ & $11.01 \pm 5.6$ & 0.197 \\
\cline { 2 - 5 } & Maximum (Gy) & $16.88 \pm 8$ & $16.87 \pm 7.5$ & 0.98 \\
\hline Left Optic nerve & Mean (Gy) & $10.73 \pm 5.3$ & $12.81 \pm 8$ & 0.333 \\
\cline { 2 - 5 } & Maximum (Gy) & $16.58 \pm 7.9$ & $16.57 \pm 8.1$ & 0.947 \\
\hline Right Cochlea & Mean (Gy) & $35.97 \pm 2.9$ & $35.89 \pm 3$ & 0.728 \\
\cline { 2 - 5 } & Maximum (Gy) & $38.78 \pm 3.1$ & $38.39 \pm 3$ & 0.392 \\
\hline Left Cochlea & Mean (Gy) & $35.59 \pm 4.4$ & $36.19 \pm 4$ & 0.414 \\
\cline { 2 - 5 } & Maximum (Gy) & $38.79 \pm 4.6$ & $38.37 \pm 4.2$ & 0.175 \\
\hline
\end{tabular}

VMAT: Volumetric modulated arc therapy

Table 3: Comparison of mean of doses calculated at brain stem i and brain stem ii in combined VMAT-CyberKnife sum plan

\begin{tabular}{llll}
\hline Parameter & Brain stem i & Brain stem ii & p value \\
\hline Maximum (Gy) & $57.55 \pm 0.42$ & $59.67 \pm 0.21$ & 0.001 \\
\hline V59 Gy (cc) & $0.15 \pm 0.14$ & $0.55 \pm 0.22$ & 0.001 \\
\hline V55.8 Gy (\%) & $16.47 \pm 4.76$ & $25.49 \pm 3.84$ & 0.001 \\
\hline Mean (Gy) & $51.6 \pm 0.93$ & $52.86 \pm 0.88$ & 0.002 \\
\hline
\end{tabular}

Table 4: Influence of different clinical features on survival rates

\begin{tabular}{|c|c|c|c|c|c|}
\hline \multirow[t]{2}{*}{ Parameter } & \multirow[t]{2}{*}{ No. $(\%)$} & \multicolumn{2}{|c|}{$\begin{array}{c}\text { Overall survival } \\
\end{array}$} & \multicolumn{2}{|c|}{ Progression-free survival } \\
\hline & & Median (95\% CI) & p value & Median (95\% CI) & p value \\
\hline \multicolumn{6}{|l|}{ Sex } \\
\hline Male & $10(66.67)$ & $45(30.3-64.2)$ & \multirow[t]{2}{*}{0.107} & $43(38.6-78.0)$ & \multirow[t]{2}{*}{0.91} \\
\hline Female & $5(33.33)$ & $60(31.1-83)$ & & $44(29.8-80.2)$ & \\
\hline \multicolumn{6}{|c|}{ Lansky play performance scale } \\
\hline $100-80$ & $7(46.67)$ & Not reached & \multirow[t]{2}{*}{0.029} & $51(38.5-55.1)$ & \multirow[t]{2}{*}{0.65} \\
\hline $70-40$ & $8(53.33)$ & $41(30.6-71)$ & & $34(30.6-81.8)$ & \\
\hline \multicolumn{6}{|l|}{ Extent of surgery } \\
\hline GTR or nGTR & $7(46.67)$ & Not reached & \multirow[t]{2}{*}{0.036} & $50(42.5-83.0)$ & \multirow[t]{2}{*}{0.055} \\
\hline Debulking or biopsy & $8(53.33)$ & $40(31.1-61.0)$ & & $36(30.5-55.2)$ & \\
\hline \multicolumn{6}{|l|}{ Histopathology } \\
\hline Grade II & $11(73.33)$ & $57(32.2-77)$ & \multirow[t]{2}{*}{0.441} & $47(30.2-70.4)$ & \multirow[t]{2}{*}{0.83} \\
\hline Grade III & $4(26.67)$ & $45(41-83)$ & & $41(36.2-80.1)$ & \\
\hline
\end{tabular}

One out of the 2 patients who had isolated PF relapse had to undergo re-excision. The other patient had extensive relapse that was deemed unresectable and was managed palliatively. Among the 5 patients who had spinal relapse, one patient had supratentorial and spinal relapse 3 years after end of RT. He underwent palliative excision of his large supra-tentorial relapsed lesion followed by irradiation of the central nervous system to $36 \mathrm{~Gy}$ in 20 fractions limiting the dose to the brain stem to $<20$ Gy in 30 fractions. Boost dose was given to the supratentorial relapsing tumor bed for an additional $18 \mathrm{~Gy}$ in 10 fractions. Three patients received palliative spinal irradiation (20 Gy in 5 fractions) for pain control. The remaining patient was managed by best supportive care.

The estimated 5-year OS was $63.6 \%$ (Figure 2). Among different clinical parameters, patients' Lanskyplay performance scale and extent of surgery had significant influence on OS (Table 4). None of the studied parameters had a significant correlation with PFS (Table $4)$.

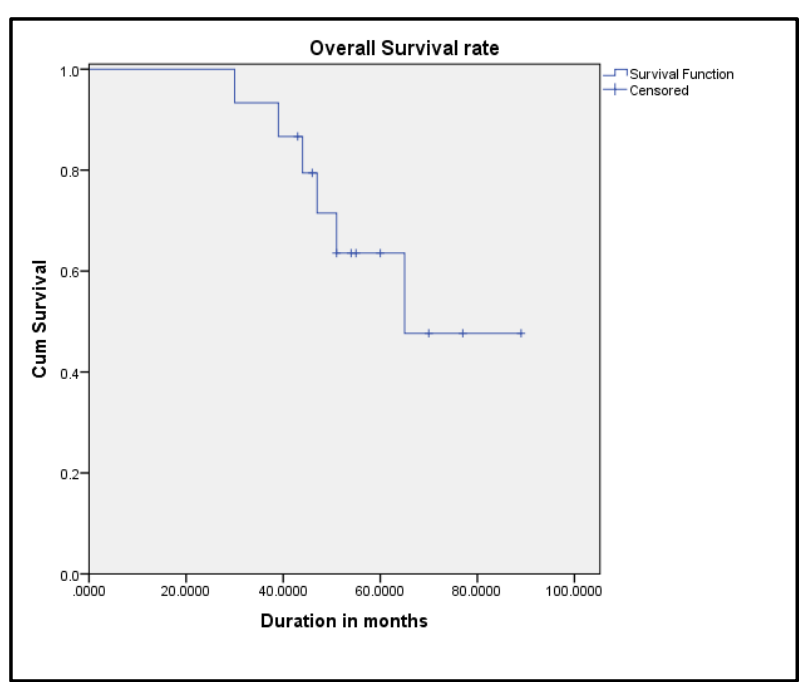

Figure 2: Overall survival curve of 15 pediatric patients with fourth ventricle ependymoma 
None of the patients developed G3/4 acute toxicities, 6 (40\%) patients developed headache, 4 (26.6\%) nausea/vomiting and $7(46.6 \%)$ grade 1 alopecia. No late toxicities in the form of radiation induced neuropathy, cognitive function impairment, vasculopathy or secondary malignancy were detected. All patients who were on steroids, were safely weaned from it during the RT course time. During follow up, we didn't need to reintroduce steroids to any of the patients except for those who developed local recurrence.

\section{DISCUSSION}

Ependymoma of the fourth ventricle is a devastating pediatric tumor. Surgery and RT formed the pillars for Children's Oncology Group (COG) ${ }^{20}$ and International Society for Pediatric Oncology (SIOP) ependymoma trials ${ }^{21}$. It is well proven that GTR is the most significant clinical predictor of superior PFS and OS ${ }^{8,12}$. Gross total resection of fourth ventricular ependymoma extending anteriorly to the brain stem and/or caudally to the spinal cord represents a real challenge to surgeons. Quite often, achieving GTR is associated with serious neurological deficits and hence not feasible. Delivery of adequate radiation doses in such critical geographical location is hindered by the tolerance of adjacent vital normal structures.

Even following GTR, most of the recent trials mandate the use of high dose localized irradiation for ependymoma. The RT regimen for SIOP-EP-II dictated that any patient $>12$ months should receive a total PD of 59.4 Gy in 33 fractions ${ }^{21}$. After initial surgery, patients in AIEOP study were treated with fractionated irradiation to a total dose of $59.4 \mathrm{~Gy}^{22}$. In COG trial ACNS0121, all patients $>18$ months received the same radiation dose. None of the included patient in this trial was $<18$ months, therefore, we applied the same dose for all patients. The entire brain stem can tolerate up to 54 Gy with $<5 \%$ risk of brain stem necrosis or neurologic toxicity. Only 1-10 cc can tolerate up to $59 \mathrm{~Gy}$, while a point dose may tolerate up to $64 \mathrm{~Gy}$. Spinal cord radiation-induced myelopathy risk is estimated to be $0.2 \%$ at 50 Gy and $5 \%$ at $59.3 \mathrm{~Gy}{ }^{23}$. Overlap of PTV and critical structures occasionally hinders achieving adequate target coverage without exceeding the tolerance threshold of the adjacent structures. CyberKnife has the advantage of online position verification and auto-correction of all translation offsets. Action level to correct for rotational shift was set to $\geq 0.3 \mathrm{~mm}$. Considering such accuracy, we didn't apply a PTV margin to the CTVii in CyberKnife plan and consequently the overlapping volumes were minimized. In the ongoing COG study, there is a required volume reduction after a dose of $54 \mathrm{~Gy}$. For doses beyond $54 \mathrm{~Gy}$, OAR constraints can be prioritized over PTV coverage ${ }^{24}$. The SIOP study recommends that for patients with PF ependymoma, the spinal cord be excluded after a total dose of $54 \mathrm{~Gy}$ and the dose to the brain stem be minimized 21 .

In the current study, VMAT plans failed to achieve satisfactory target coverage without violation of brain stem tolerance dose. Using combined techniques, we managed to cover $95.09 \pm 0.04 \%$ of the CTVii with $95 \%$ of the PD without violating the OARs tolerance doses. The sharp tumor-brain parenchyma interface and the rapid dose decline at the edge of CTVii with CyberKnife improved the dose distribution. Delivering $1.8 \mathrm{~Gy}$ per fraction was possible with beam-on time (12-15 min). In some GA patients, we accepted CyberKnife plans with marginally suboptimal dose distribution using a smaller number of beams to reduce the treatment time. This marginal advantage of combined plans over VMAT plans should be weighed against the more complex and costly course of CyberKnife plans, and should be investigated in large clinical trials to prove its clinical benefit.

Variable studies discussed the use of stereotactic radiosurgery and fractionated stereotactic radiotherapy in ependymoma cases ${ }^{25-27}$. Kano and colleagues reported the results of salvaging recurrent/residual ependymoma cases post-surgical excision and RT using stereotactic radiosurgery ${ }^{25}$. In their analysis, the 5-year OS rate for pediatric patients was $38.5 \%$ and patients with age $<18$ years had significantly worse local control rate compared to older patients $(\mathrm{p}=0.014)$. Proton therapy was recently used in the treatment of pediatric ependymoma 28, 29. It demonstrated comparable local control and survival rates when compared to the published photon studies. In Carmen and colleagues' study, the 5-year local control rate and OS rate were $78 \%$ and $84 \%$ compared to $80 \%$ and $63.6 \%$ in our study; respectively ${ }^{29}$.

There was an average of 7.2 weeks lapse between phase I and phase II planning MRI scans. This period is usually adequate for partial resolution of the postoperative changes and further collapse of the tumor bed. These anatomical changes contributed to the relative posterior displacement of the brain stem closer to the tumor bed and the high dose region (Figure 3).

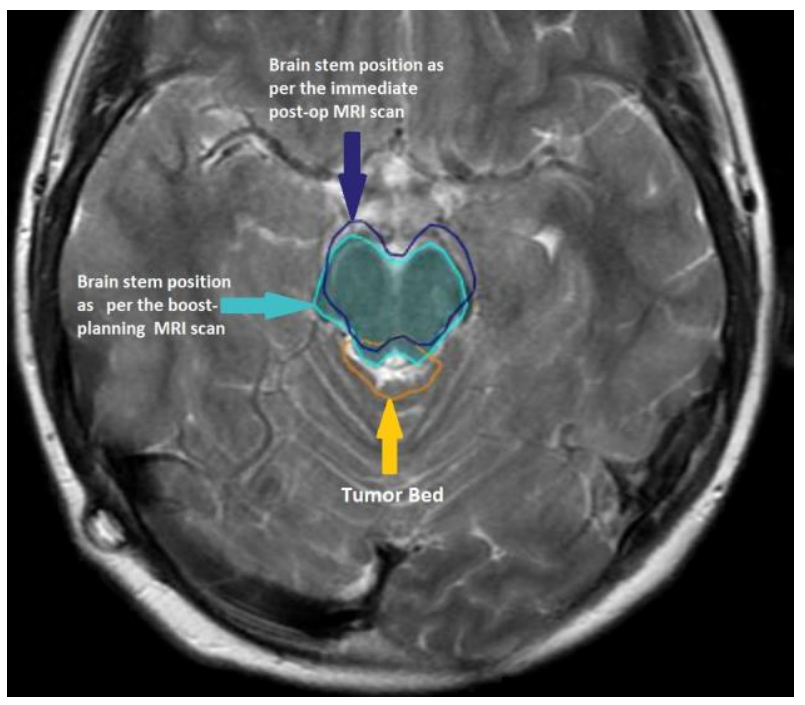

Figure 3: Brain stem position displacement prior to boost phase

Dose calculated at brain stem location in the boost planning-MRI (BSii) was relatively higher compared to its immediate post-operative location (BSi). Though the difference is not large, this difference is significant and may be critical in some cases. We believe that planning MRI brain scan should be done within 2 weeks before 
starting phase I. In addition, a new MRI scan should be done within 2 weeks prior to boost phase to avoid brain stem and/or tumor bed displacement.

The fact that local failure may represent up to $80 \%$ of the relapsing cases emphasizes the value of GTR to outcome $^{6,30-32}$. In our work, large sized lesions, highly vascular tumors or extension to the brain stem were the main reasons that precluded attaining GTR in some cases. In the current study, only $33.3 \%$ of patients had GTR compared to $83 \%$ in Merchant et al ${ }^{8}, 69 \%$ in Massimino et al ${ }^{22}$ and $63.3 \%$ in Marinoff et al ${ }^{12}$ studies. Though local control rate was $80 \%$, the estimated 5-year OS and PFS rates in our study were unsatisfactory representing $63.6 \%$ and $53.3 \%$, respectively. Merchant et al reported 7 -year OS and PFS rates of $81 \%$ and $69.1 \%{ }^{8}$. Marinoff et al reported a 5-year OS and PFS rate of $67 \pm 5 \%$ and $39 \pm 5 \%$, respectively ${ }^{12}$.

The relatively low GTR rate in our study possibly contributed to the relatively lower survival rates despite post-operative irradiation. Though not consistent in all studies, the infratentorial location of tumors might be another contributing factor for the relatively poor outcome $^{22,33}$. One of the drawbacks in our study was the lack of molecular classification of the disease. Possible deletion of CDKN2A, gain of chromosome 1 and/or presence of RELA fusion event in our patients might have contributed to the relatively poor OS and PFS. Another limitation of the current study is that our treatment protocol lacks the use of chemotherapy and second look surgery prior to irradiation in cases where GTR was not feasible at initial surgery. After a median follow up period of 51 months, no radiation-induced neuropathy or cognitive function impairment were reported. Though it is expected following such conservative constrains adopted in this study, a longer follow up period is needed to exclude the development of any late sequelae particularly second malignancies.

\section{Conclusion}

VMAT plans failed to achieve satisfactory target coverage without violation of brain stem tolerance dose. Conversely, using a combination of VMAT and CyberKnife technique, it was feasible to reach adequate dose coverage of ependymoma of the fourth ventricle without exceeding the tolerance of the adjacent critical structures. The adopted technique was tolerable with no recorded long-term radiation-induced toxicities.

\section{FUNDING}

The authors did not receive funding for this study.

\section{CONFLICT OF INTEREST}

The authors have no conflict of interest to declare.

\section{REFERENCES}

1. Chamberlain MC. Ependymomas. Curr Neurol Neurosci Rep. 2003; 3(3):193-199.

2. Shim KW, Kim DS, Choi JU. The history of ependymoma management. Childs Nerv Syst. 2009; 25(10):1167-1183
3. Hamilton RL, Pollack IF. The molecular biology of ependymomas. Brain Pathol. 1997; 7(2): 807-22.

4. Ben Ammar CN, Kochbati L, Frikha H, Gargouri W, Benna $\mathrm{F}$, Besbes $\mathrm{M}$, et al. Primitive intracranial ependymomas. Salah-Azaîz institute experience. Cancer Radiother. 2004; 8(2): 75-80.

5. Merchant TE, Fouladi M. Ependymoma: new therapeutic approaches including radiation and chemotherapy. J Neurooncol. 2005; 75(3): 287-299.

6. Mansur DB, Perry A, Rajaram V, Michalski JM, Park TS, Leonard JR, et al. Postoperative radiation therapy for grade II and III intracranial ependymoma. Int J Radiat Oncol Biol Phys. 2005; 61(2): 387-391.

7. Shu HK, Sall WF, Maity A, Tochner ZA, Janss AJ, Belasco JB, et al. Childhood intracranial ependymoma: twenty-year experience from a single institution. Cancer. 2007; 110(2): 432-441.

8. Merchant TE, Li C, Xiong X, Kun LE, Boop FA, Sanford RA. Conformal radiotherapy after surgery for paediatric ependymoma: a prospective study. Lancet Oncol. 2009; 10(3): 258-266.

9. Oya N, Shibamoto Y, Nagata Y, Negoro Y, Hiraoka M. Postoperative radiotherapy for intracranial ependymoma: analysis of prognostic factors and patterns of failure. $\mathrm{J}$ Neurooncol. 2002; 56(1): 87-94.

10. van Veelen-Vincent ML, Pierre-Kahn A, Kalifa C, SainteRose C, Zerah $\mathrm{M}$, Thorne $\mathrm{J}$, et al. Ependymoma in childhood: prognostic factors, extent of surgery, and adjuvant therapy. J Neurosurg. 2002; 97(4): 827-835.

11. Robertson PL, Zeltzer PM, Boyett JM, Rorke LB, Allen JC, Geyer JR, et al. Survival and prognostic factors following radiation therapy and chemotherapy for ependymomas in children: a report of the Children's Cancer Group. J Neurosurg. 1998; 88(4): 695-703.

12. Marinoff AE, Ma C, Guo D, Snuderl M, Wright KD, Manley PE, et al. Rethinking childhood ependymoma: a retrospective, multi-center analysis reveals poor long-term overall survival. J Neurooncol. 2017; 135(1): 201-211.

13. Mack SC, Witt H, Wang X, Milde T, Yao Y, Bertrand KC, et al. Emerging insights into the ependymoma epigenome. Brain Pathol. 2013; 23(2): 206-209.

14. Gajjar A, Pfister SM, Taylor MD, Gilbertson RJ. Molecular insights into pediatric brain tumors have the potential to transform therapy. Clin Cancer Res. 2014; 20(22): 56305640.

15. Pajtler KW, Witt H, Sill M, Jones DT, Hovestadt V, Kratochwil F, et al. Molecular classification of ependymal tumors across all CNS compartments, histopathological grades, and age groups. Cancer Cell. 2015; 27(5): 728-743.

16. Seiler G, De Vol E, Khafaga Y, Gregory B, Al-Shabanah $\mathrm{M}$, Valmores A, et al. Evaluation of the safety and efficacy of repeated sedations for the radiotherapy of young children with cancer: a prospective study of 1033 consecutive sedations. Int J Radiat Oncol Biol Phys. 2001; 49(3): 771783.

17. Trotti A, Byhardt R, Stetz J, Gwede C, Corn B, Fu K, et al. Common Toxicity Criteria: Version 2.0. an improved reference for grading the acute effects of cancer treatment: impact on radiotherapy. Int J Radiat Oncol Biol Phys. 2000; 47(1): 13-47.

18. Louis DN, Perry A, Reifenberger G, von Deimling A, Figarella-Branger D, Cavenee WK, et al. The 2016 World Health Organization Classification of Tumors of the Central Nervous System: a summary. Acta Neuropathol. 2016; 131(6): 803-820.

19. Lansky SB, List MA, Lansky LL, Ritter-Sterr C, Miller DR. The measurement of performance in childhood cancer patients. Cancer. 1987; 60(7): 1651-1656.

20. Merchant TE. Observation or radiation therapy and/or chemotherapy and second surgery in treating children who 
have undergone surgery for ependymoma (ClinicalTrials.gov Identifier: NCT00027846). Available from: https://clinicaltrials.gov/ct2/show/NCT00027846? term $=$ nct00027846\&rank $=1$. 2001. Last accessed: 5 March 2020.

21. Frappaz D. An international clinical program for the diagnosis and treatment of children with ependymoma (SIOP-EP-II) (ClinicalTrials.gov Identifier: NCT02265770). Available from: https://clinicaltrials.gov/ct2/ show/ NCT02265770?term $=$ nct02265770\&rank $=1$. 2014. Last accessed: 5 March 2020.

22. Massimino M, Miceli R, Giangaspero F, Boschetti L, Modena P, Antonelli M, et al. Final results of the second prospective AIEOP protocol for pediatric intracranial ependymoma. Neuro Oncol. 2016; 18(10): 1451-1460.

23. Marks LB, Yorke ED, Jackson A, Ten Haken RK, Constine LS, Eisbruch A, et al. Use of normal tissue complication probability models in the clinic. Int J Radiat Oncol Biol Phys. 2010; 76(3 Suppl): S10-19.

24. Smith AA. Maintenance chemotherapy or observation following induction chemotherapy and radiation therapy in treating younger patients with newly diagnosed ependymoma (ClinicalTrials.gov Identifier: NCT01096368). Available from: https://clinicaltrials.gov/ct2/show/

NCT01096368?term=nct01096368\&rank=1. 2010. Last accessed: 5 March 2020.

25. Kano H, Su YH, Wu HM, Simonova G, Liscak R, CohenInbar $\mathrm{O}$, et al. Stereotactic radiosurgery for intracranial ependymomas: an international multicenter study. Neurosurgery. 2019; 84(1): 227-234.
26. Liu AK, Foreman NK, Gaspar LE, Trinidad E, Handler MH. Maximally safe resection followed by hypofractionated reirradiation for locally recurrent ependymoma in children. Pediatr Blood Cancer. 2009; 52(7): 804-807.

27. Lobón MJ, Bautista F, Riet F, Dhermain F, Canale S, Dufour C, et al. Re-irradiation of recurrent pediatric ependymoma: modalities and outcomes: a twenty-year survey. Springerplus. 2016; 5(1): 879.

28. Indelicato DJ, Bradley JA, Rotondo RL, Nanda RH, Logie N, Sandler ES, et al. Outcomes following proton therapy for pediatric ependymoma. Acta Oncol. 2018; 57(5): 644-648.

29. Ares C, Albertini F, Frei-

Welte M, Bolsi A, Grotzer MA, Goitein G, et al. Pencil beam scanning proton therapy for pediatric intracranial ependymoma. J Neurooncol. 2016; 128(1): 137-145.

30. Healey EA, Barnes PD, Kupsky WJ, Scott RM, Sallan SE, Black PM, et al. The prognostic significance of postoperative residual tumor in ependymoma. Neurosurgery. 1991; 28(5): 666-671; discussion 671-672.

31. McLaughlin MP, Marcus RB Jr, Buatti JM, McCollough WM, Mickle JP, Kedar A, et al. Ependymoma: results, prognostic factors and treatment recommendations. Int $\mathbf{J}$ Radiat Oncol Biol Phys. 1998; 40(4): 845-850.

32. Timmermann B, Kortmann RD, Kühl J, Meisner C, Slavc I, Pietsch T, et al. Combined postoperative irradiation and chemotherapy for anaplastic ependymomas in childhood: results of the German prospective trials HIT 88/89 and HIT 91. Int J Radiat Oncol Biol Phys. 2000; 46(2): 287-295.

33. Horn B, Heideman R, Geyer R, Pollack I, Packer R, Goldwein $\mathrm{J}$, et al. A multi-institutional retrospective study of intracranial ependymoma in children: identification of risk factors. J Pediatr Hematol Oncol. 1999; 21(3): 203-211. 